\title{
Front Matter: Volume 11327
}

, "Front Matter: Volume 11327," Proc. SPIE 11327, Optical Microlithography XXXIII, 1132701 (13 April 2020); doi: 10.1117/12.2570937

SPIE. Event: SPIE Advanced Lithography, 2020, San Jose, California, United States 


\section{PROCEEDINGS OF SPIE}

\section{Optical Microlithography XXXIII}

Soichi Owa

Mark C. Phillips

Editors

25-26 February 2020

San Jose, California, United States

Sponsored and Published by

SPIE 
The papers in this volume were part of the technical conference cited on the cover and title page. Papers were selected and subject to review by the editors and conference program committee. Some conference presentations may not be available for publication. Additional papers and presentation recordings may be available online in the SPIE Digital Library at SPIEDigitalLibrary.org.

The papers reflect the work and thoughts of the authors and are published herein as submitted. The publisher is not responsible for the validity of the information or for any outcomes resulting from reliance thereon.

Please use the following format to cite material from these proceedings:

Author(s), "Title of Paper," in Optical Microlithography XXXIII, edited by Soichi Owa, Mark C. Phillips, Proceedings of SPIE Vol. 11327 (SPIE, Bellingham, WA, 2020) Seven-digit Article CID Number.

ISSN: 0277-786X

ISSN: 1996-756X (electronic)

ISBN: 9781510634213

ISBN: 9781510634220 (electronic)

Published by

SPIE

P.O. Box 10, Bellingham, Washington 98227-0010 USA

Telephone +1 3606763290 (Pacific Time) · Fax +1 3606471445

SPIE.org

Copyright (C 2020, Society of Photo-Optical Instrumentation Engineers.

Copying of material in this book for internal or personal use, or for the internal or personal use of specific clients, beyond the fair use provisions granted by the U.S. Copyright Law is authorized by SPIE subject to payment of copying fees. The Transactional Reporting Service base fee for this volume is $\$ 18.00$ per article (or portion thereof), which should be paid directly to the Copyright Clearance Center (CCC), 222 Rosewood Drive, Danvers, MA 01923. Payment may also be made electronically through CCC Online at copyright.com. Other copying for republication, resale, advertising or promotion, or any form of systematic or multiple reproduction of any material in this book is prohibited except with permission in writing from the publisher. The CCC fee code is 0277$786 \times / 20 / \$ 18.00$

Printed in the United States of America.

Publication of record for individual papers is online in the SPIE Digital Library.

\section{SPIE. DIGITAL}

Paper Numbering: Proceedings of SPIE follow an e-First publication model. A unique citation identifier (CID) number is assigned to each article at the time of publication. Utilization of CIDs allows articles to be fully citable as soon as they are published online, and connects the same identifier to all online and print versions of the publication. SPIE uses a seven-digit CID article numbering system structured as follows:

- The first five digits correspond to the SPIE volume number.

- The last two digits indicate publication order within the volume using a Base 36 numbering system employing both numerals and letters. These two-number sets start with 00, 01, 02, 03, 04, 05, 06, 07, 08, 09, 0A, OB ... 0Z, followed by 10-1Z, 20-2Z, etc. The CID Number appears on each page of the manuscript. 


\section{Contents}

$\begin{array}{ll}\text { vii } & \text { Authors } \\ \text { ix } & \text { Conference Committee }\end{array}$

KEYNOTE SESSION AND LATEST TOPIC

1132705 Pixelated mask optimization on quantum computers [11327-3]

MACHINE LEARNING AND COMPUTATIONAL LITHOGRAPHY I

1132706 Establishing fast, practical, full-chip ILT flows using machine learning (Invited Paper) [1 1327-4]

1132707 Mask synthesis using machine learning software and hardware platforms (Invited Paper) [11327-5]

1132709 Model based CAOPC flow for memory chips to improve performance and consistency of RET solutions [11327-41]

11327 OA Physics based feature vector design: a critical step towards machine learning based inverse lithography [1 1327-7]

MACHINE LEARNING AND COMPUTATIONAL LITHOGRAPHY II

$11327 \mathrm{OB} \quad$ Accurate etch modeling with massive metrology and deep-learning technology [11327-8]

11327 OC SRAF printing prediction using artificial neural network [11327-9]

11327 OD Improving ORC methods and hotspot detection with the usage of aerial images metrology [11327-10]

11327 OF Fast all-angle Mask 3D for ILT patterning [11327-12]

PROCESS CONTROL, RESIST MODELING

11327 OG Resist shrinkage during development: rigorous simulations and first compact model for OPC [1 1327-13]

$11327 \mathrm{OH} \quad$ Compact resist model using single convolution kernel [1 1327-14] 
11327 Ol Rigorous simulation of implant resist on topographic wafer [11327-15]

11327 OJ Rigorous vase data fitting for ultrathin films [11327-16]

11327 OK TrueMask ILT MWCO: full-chip curvilinear ILT in a day and full mask multi-beam and VSB writing in $12 \mathrm{hrs}$ for 193i [11327-17]

LITHOGRAPHY EQUIPMENT, FOCUS CONTROL

11327 OM Divided spectrum illumination for high resolution flat panel display exposure tools [1 1327-19]

$11327 \mathrm{ON} \quad$ Investigating the fine line lithography process on the large-size organic panels for advanced packaging and development [1 1327-20]

1132700 Accuracy improvement in advance lithography focus control [1 1327-21]

DUV AND EUV MATCHING

11327 OS Holistic alignment approach for on-product overlay improvement on DUV lithography process with combined solutions [11327-24]

11327 OT A novel projection lens manipulator for high frequent overlay tuning [1 1327-25]

OVERLAY AND CD CONTROL

11327 OU Advanced high order field-to-field modeling for wafer edge control [1 1327-26]

11327 OV Application of intra-field alignment to reduce wafer-to-wafer variation [1 1327-27]

11327 OW Novel overlay correction using inline alignment station (iAS) for scanner [1 1327-28]

11327 OX Improvement of SADP CD control in 7nm BEOL application [1 1327-48]

11327 OY Through-the-mask (TM) optical alignment for high volume manufacturing nanoimprint lithography systems [1 1327-30]

POSTER SESSION

$113270 Z$ Design and fabrication of UVLED array aligner for proximity and soff contact exposure [1 1327-31]

iv 
$1132711 \quad$ Proteus SMO for process window improvement [11327-33]

1132713 Approach for lightsource utilization improvement by extending Preventive Maintenance (PM) cycle along with performance monitoring feature [1 1327-35]

1132716 Comparison of different lithographic source optimization methods based on compressive sensing [11327-38]

1132717 Imaging performance enhancement by improvements of spectral performance stability and controllability on the cutting-edge [11327-39]

1132719 Fast algorithm of the scanning lithographic metrics based on a quadratic imaging model and an integral transfer function [1 1327-42]

$113271 \mathrm{~A}$ Target recovery process optimization to improve image based overlay performance for critical recording head manufacturing processes [11327-43]

$113271 \mathrm{~B} \quad$ KrF excimer laser-based patterning system for dual applications in both lithography and ablation [1 1327-45]

11327 1C Evaluating and correcting pattern variability induced by OPC within regular array layout [1 1327-46] 
Proc. of SPIE Vol. 11327 1132701-6 Downloaded From: https://www.spiedigitallibrary.org/conference-proceedings-of-spie on 25 Apr 2023
Terms of Use: https://www.spiedigitallibrary.org/terms-of-use 


\section{Authors}

Numbers in the index correspond to the last two digits of the seven-digit citation identifier (CID) article numbering system used in Proceedings of SPIE. The first five digits reflect the volume number. Base 36 numbering is employed for the last two digits and indicates the order of articles within the volume. Numbers start with 00, 01, 02, 03, 04, 05, 06, 07, 08, 09, 0A, 0B...0Z, followed by 10-1Z, 20-2Z, etc.

Ando, Miwako, $0 \mathrm{M}$

Arce, Gonzalo R., 16

Bald, Holger, OU, OV

Bellmann, Enrico, OV

Böcker, Paul, OS

Bouaricha, Ali, OK

Bouman, Wim, OT

Bowser, Aaron, 1A

Braam, Kyle, 06

Buhl, Stefan, OU

Bushida, Satoru, 17

Buttgereit, Ute, OD

Cecil, Thomas, 06

Chang, Jonathan, $\mathrm{OX}$

Chen, Kun-Yuan, 11

Chen, Rui, 16

Chen, Shoumian, 0A

Chen, Xiuguo, 19

Chong, Nui, OX

Collins, Darin, 0J

Dam, Thuc, 11

de Boeij, Wim, OT

Depre, L., 1C

Dineen, Cassidy, 00

Ding, Hua, 11

Dong, Lisong, 16

Emer, Wolfgang, ОT

Eło, Shigeru, OW

Fahrni, Francis, OT

Fan, Yongfa, OB

Fan, Zhiqiang, OJ

Feng, Mu, OB

Feng, Yaobin, 09

Fujimura, Aki, OF, OK

Gao, Ying, OB

Gardin, C., 1C

Granik, Yuri, OG

Ha, Hyunjun, OV

Habets, Boris, OU, OV

Hakko, Manabu, OM

Han, Sang-Jun, OS

Hany Mousa, Sherif, 09

Hao, Xueli, 00

Hayashi, Nozomu, OY

Hirai, Shinichirou, OY

Hisamura, Toshiyuki, OX

Hoeer, Tobias, OU, OV

Hoppe, Wolfgang, 01

Hsu, Chris, 00
Hsu, Poya, 00

$\mathrm{Hu}$, Hongmei, OB

$\mathrm{Hu}$, Sophia, 13

Huang, Jiun-Woei, $0 Z$

Igarashi, Miwa, 17

lino, Satoshi, OY

Itani, Toshiro, 1B

Iwai, Toshiki, OY

Izumi, Nozomu, OM

Jayaram, Srividya, 09

Ji, Liang, OB

Jia, Jianjun, 11

Jiang, Hao, 19

Katou, Masahide, 17

Kawashima, Tohru, OY

Khaira, Daman, OG

Kikuchi, Takahisa, OW

Kim, Cheolkyun, OC

Kim, Gwang-Gon, OS

Kim, Jangsun, OV

Kim, Jung-Hwan, OS

Kim, Jun-Yeob, OS

Kim, Kyong-Seok, OS

Kim, Seop, OV

Kim, Sungho, OC

Kim, Wan-Soo, OU

Kim, Young Ki, 00

Kimura, Taiki, $\mathrm{OH}$

Klinkhamer, Friso, OT

Komaki, Takamitsu, OY

Küchler, Bernd, 0 I

Kurosu, Akihiko, 17

Kwon, Yonghwi, OC

La Greca, R., 1C

LaCour, Pat, 09

Lamb, James, 0J

Lambregts, Cees, OS

Lan, Andy, 11

Lang, Jun, $O B$

Lee, Chih-Jie, 11

Lee, Seonho, OV

Li, Chen, OA

Li, Ming, OB

Limmer, Tim, 0J

Lin, Cheng-Shuan, 11

Lin, Qi, OX

Liu, Jing Jing, 11

Liu, Liang, $\mathrm{OB}$

Liu, Peng, 07 
Liu, Shiyuan, 19

Liu, Yi, $1 \mathrm{~A}$

Liu, Zhunhua, OB

Lomtscher, Patrick, OU

Lowes, Joyce, 0J

Lu, Yifei, OB

Ma, Jigang, OS

Ma, Won-Kwang, oS

$\mathrm{Ma}, \mathrm{Xu}, 16$

Matsumoto, Takahiro, $0 Y$

Matsunawa, Tetsuaki, $\mathrm{OH}$

Meyer, Mike, OF

Mimotogi, Shoji, $\mathrm{OH}$

Minoda, Ken, OY

Mishima, Kazuhiko, oY

Miyaharu, Takafumi, OY

Miyamoto, Hirotaka, 17

Mizoguchi, Hakaru, 17

Morgenfeld, Bradley, 00

Morita, Naoki, OW

Moriya, Masato, 17

Nagano, Kouhei, OM

Niewczas, Mariusz, OK

Oga, Toshihiro, 13

Ohta, Takeshi, 17

Okudaira, Yosuke, 05

Okutomi, Akira, OW

Omran, Ahmed, 06

Pan, Bingyang, $O B$

Pang, Linyong (Leo), OF, OK

Park, Chan-Ha, OS

Park, Sang-Jun, OS

Pearman, Ryan, OF, OK

Pena, J. N., IC

Peng, Xiang, $\mathrm{OB}$

Pollak, Thilo, OT

Pomerantsev, Michael, OK

Poonawala, Amyn, 06

Rathi, Ashutosh, 09

Saitou, Takashi, 17

Santillan, Julius Joseph, 1B

Sato, Futoshi, 13

Schatz, Jirka, 이

Sha, LU, OK

Sheng, Xichen, OB

Shi, Xuelong, OA

Shi, Yating, 19

Shin, Youngsoo, OC

Shu, Jason, 06

Sohara, Naoya, ON

Stock, Hans-Juergen, 16

Stöłzel, Christian, OD

Su, Bo, OK

Sugimoto, Ayako, OW

Sungaver, E., 1C

Suzuki, Kanji, OM

Takahashi, Kazuhiro, OY

Takahashi, Ryotaro, ON

Takamatsu, Hirosuke, ON
Takasaki, Koichi, OM

Taki, Tomokazu, OY

Tanaka, Hiroshi, 17

Tanaka, Satoshi, 17

Thaler, Thomas, OD

Thüring, Bernd, OT

Tian, $\mathrm{YU}, \mathrm{OB}$

Tsamados, Dimitrios, 0

Tsiachris, Sotirios, OS

Tsushima, Hiroaki, 17

Uemori, Nobutaka, 1B

Ungar, P. Jeffrey, OF, OK

Unno, Yasuyuki, OY

Vandam, Clark, 06

Wang, Jinze, $O B$

Wang, Kechang, OK

Wang, Weijun, OB

Wang, Zhiqiang, 16

Wei, Yayi, 16

Weisbuch, Francois, OD

WU, Ying-chen, OB

Xiao, Yanjun, $O B$

Xie, Qian, OB

$X U$, Ting Ting, 11

Yamaoka, Hiroshi, 1B

Yamazaki, Taku, 13

Yan, Guanyong, OB

Yang, Dongyue, 00

Yang, Jinho, OC

Yang, Richer, 11

Yang, Terrance, OB

Yang, Zhiyong, 19

Yao, Jun, 09

Yao, Shuxin, $O B$

Yao, Yueliang, OB

Yashiki, Satoshi, 05

Ye, Shawn, OJ

Yi, Chunyan, OB

Yu, Dan, IA

Yu, Henry, OF

Yu, Miao, OS

Yuan, Wei, OB

Zeuner, Thomas, OD

Zhang, Lei, 09

Zhao, Qian, OB

Zhao, Yiqiong, $O B$

Zhao, Yuhang, OA, OB

Zheng, Zhenguo, 09

Zhu, Zhimin, OJ 


\section{Conference Committee}

Symposium Chairs

Will Conley, Cymer, LLC (United States)

Kafai Lai, IBM Thomas J. Watson Research Center (United States)

Conference Chair

Soichi Owa, Nikon Corporation (Japan)

Conference Co-chair

Mark C. Phillips, Intel Corporation (United States)

Conference Program Committee

Jack Chen, NanoPatterning Technology Company, Ltd. (Taiwan)

Will Conley, Cymer, An ASML company (United States)

Carlos Fonseca, Tokyo Electron America, Inc. (United States)

Bernd Geh, Carl Zeiss SMT GmbH (United States)

Yuri Granik, Mentor Graphics Corporation (United States)

Harsha Grunes, Intel Corporation (United States)

Toshiyuki Hisamura, Xilinx, Inc. (United States)

Stephen D. Hsu, ASML San Jose (United States)

Young Seog Kang, SAMSUNG Electronics Company, Ltd.

(Korea, Republic of)

Sachiko Kobayashi, Toshiba Corporation (Japan)

Jongwook Kye, SAMSUNG Electronics Company, Ltd.

(Korea, Republic of)

Kafai Lai, IBM Corporation (United States)

Ken-Ichiro Mori, Canon Inc. (Japan)

John S. Petersen, IMEC (United States)

Daniel Sarlette, Infineon Technologies Dresden (Germany)

Kunal N. Taravade, Synopsys, Inc. (United States)

Edita Tejnil, Mentor Graphics Corporation (United States)

Geert Vandenberghe, IMEC (Belgium)

Reinhard Voelkel, SUSS MicroOptics SA (Switzerland)

Uwe D. Zeitner, Fraunhofer-Institut für Angewandte Optik und Feinmechanik (Germany) 


\section{Session Chairs}

1 Keynote Session and Latest Topic

Soichi Owa, Nikon Corporation (Japan)

Mark C. Phillips, Intel Corporation (United States)

2 Machine Learning and Computational Lithography I

Kunal N. Taravade, Synopsys, Inc. (United States)

Edita Tejnil, Mentor Graphics Corporation (United States)

3 Machine Learning and Computational Lithography II

Harsha Grunes, Intel Corporation (United States)

Stephen D. Hsu, ASML San Jose (United States)

4 Process Control, Resist Modeling

Geert Vandenberghe, imec (Belgium)

Carlos Fonseca, Tokyo Electron America, Inc. (United States)

5 Lithography Equipment, Focus Control

Reinhard Voelkel, SUSS MicroOptics SA (Switzerland)

Ken-Ichiro Mori, Canon Inc. (Japan)

6 DUV and EUV Matching

Bernd Geh, Carl Zeiss SMT GmbH (United States)

Young Seog Kang, SAMSUNG Electronics Company, Ltd.

(Korea, Republic of)

7 Overlay and CD control

Toshiyuki Hisamura, Xilinx, Inc. (United States)

Jack Chen, NanoPatterning Technology Company, Ltd. (Taiwan) 